\title{
Validação da Escala de Bem-Estar Espiritual para pacientes hospitalizados no período pré-operatório
}

\author{
Validation of the Spiritual Well-Being Scale for \\ hospitalized patients in the preoperative period \\ Eduardo Tavares Gomes', Simone Maria Muniz da Silva Bezerra'
}

\section{RESUMO}

Objetivo: Validar o uso da Escala de Bem-Estar Espiritual para pacientes em internamento no pré-operatório. Métodos: Estudo de validação da versão brasileira da Escala de Bem-Estar Espiritual para aplicação em pacientes em internamento no período pré-operatório, realizado entre os meses de janeiro e abril de 2017, nas enfermarias de um hospital universitário especializado em cardiologia clínica e cirúrgica. Realizou-se análise fatorial exploratória por rotação ortogonal do tipo Varimax. Foram avaliadas a fidedignidade e a consistência interna e as correlações entre os itens e o total. Resultados: A amostra com 174 participantes continha a razão de 8,7 pessoas por item (variável), considerada adequada ao propósito da análise fatorial $(\mathrm{KMO}=0,87)$, e o Teste de Esferacidade de Bartlett's foi significativo $\left(\chi^{2}=1401,67 ; \mathrm{p}<\right.$ $0,01)$. Os dois fatores rotacionados ficaram constituídos como a proposta inicial, com os itens ímpares representando a subescala de Bem-Estar Religioso $(a=0,84)$ e os pares representando a subescala de Bem-Estar Existencial $(a=0,71)$, com correlação entre os dois fatores de $r=0,47$. A análise das cargas fatoriais para a subescala de Bem-Estar Religioso apresentou distribuição próxima à da validação inicial, com todos os itens com carga fatorial $>0,3$. Na avaliação dos itens da subescala de Bem-Estar Existencial, três itens alcançaram escores menores que 0,3 de carga fatorial. Conclusão: A Escala de Bem-Estar Espiritual mostrou-se útil para avaliar o construto a que se propõe quando aplicada a pacientes internados no período pré-operatório.

Psicometria, estudos de validação, espiritualidade, adaptação psicológica.

\begin{abstract}
Objective: To validate the use of the Spiritual Well-being Scale for hospitalized patients in the preoperative period. Methods: Validation study of the Brazilian version of the Spiritual WellBeing Scale for use in hospitalized patients in the preoperative period, performed between January and April 2017, in the wards of a university hospital specialized in clinical and surgical cardiology. Exploratory factorial analysis was performed by Varimax orthogonal rotation. The reliability and internal consistency and the correlations between the items and the total were evaluated. Results: The sample with 174 participants contained a ratio of 8.7 people per item (variable), considered adequate for the purpose of the factorial analysis $(\mathrm{KMO}=0.87)$ and the
\end{abstract}

1 Universidade de Pernambuco (UPE), Programa Associado de Pós-graduação em Enfermagem, Recife, PE, Brasil.

Recebido em

$11 / 11 / 2017$

Aprovado em

29/3/2018

DOI: 10.1590/0047-2085000000199
Endereço para correspondência: Eduardo Tavares Gomes Universidade de Pernambuco (UPE)

Rua Arnóbio Marques, 310, Santo Amaro

50100-130 - Recife, PE, Brasil

E-mail: edutgs@hotmail.com 


\section{Keywords}

Psychometrics, validation

studies, spirituality,

psychological adaptation.
Bartlett's Sphericity Test was significant $\left(\chi^{2}=1401.67, \mathrm{p}<0.01\right)$. The two rotated factors were constituted as the initial proposal, with the odd items representing the subscale Religious Well-being $(a=0.84)$ and the pairs, representing the subscale of Existential Well-being ( $a=$ 0.71 ), with correlation between the two factors of $r=0.47$. The analysis of the factorial loads for the subscale of Religious Wellbeing presented distribution close to the initial validation, with all items with a factorial load $>0.3$. In the evaluation of Existential Well-being subscale items, three items reached scores less than 0.3 factorial load. Conclusion: The Spiritual WellBeing Scale was proved useful to evaluate the construct that is proposed when applied to hospitalized patients in the preoperative period.

\section{INTRODUÇÃO}

Desde a conferência de Alma-Ata, em 1970, abriu-se campo para discussões do que seria saúde, conceituada a partir de um estado de completo bem-estar biopsicossocial. A partir daí, em todo o mundo, tal referencial muniu pesquisadores e profissionais para outras construções, contrapondo-se ao paradigma biomédico e possibilitando o aprimoramento de um termo que surgia na mesma época e que refletia bem o conceito de saúde proposto: integralidade'.

Na Assembleia Mundial de Saúde, em 1983, surgiram as discussões iniciais a favor da inclusão de uma dimensão não material ou espiritual de saúde. As discussões tomaram grande proporção, chegando a, durante a Assembleia de 1998, ter sido proposta uma emenda para a constituição da Organização Mundial de Saúde (OMS) para modificar o conceito clássico de saúde para com a inclusão da dimensão espiritual $^{2}$. Contudo, essa ampliação do conceito de saúde, vigente desde 1948 na carta de princípios da OMS, não foi aprovada de pronto, decidindo-se por ampliar mais as discussões acerca e continuar o debate, o que reflete a necessidade de pesquisas nessa área ${ }^{3}$.

A dimensão espiritual da saúde é a consideração da verdadeira essência do ser. A dimensão física reflete ausência de doenças, mas o conceito de saúde vai além. A dimensão psicológica considera a relação do individuo consigo próprio; a social, a relação com o outro. No entanto, a dimensão espiritual amplia essa compreensão para ver saúde também como harmonia do ser, em essência, com sua transcendência, seus valores ético-morais, sua integralidade. Essa harmonia de dimensões físicas e metafísicas reflete em saúde, promovendo bem-estar, adoção de práticas e estilos de vida mais saudáveis e melhor autocuidado ${ }^{4}$. Uma pessoa pode ser saudável psicofiscamente, mas frustrada existencialmente, assim como pode ter problemas psicofísicos e ser saudável do ponto de vista existencial-espirituals.

Pensar no bem-estar espiritual requer uma visão expandida da pessoa e do que significa ser humano, que, apesar de plenamente corporizado, é mais do que o corpo físico; é alma corporizada; uma consciência transpessoal, transcendente e evolutiva ${ }^{6}$. O conceito de saúde na perspectiva transpessoal está colocado na harmonia entre mente, corpo e alma, corre- lacionando, assim, três dimensões em que o bem-estar pode se expressar como indicador de saúde: mental (psicológico, emocional, social), corporal (físico e ecológico) e da alma (espiritual) ${ }^{7}$.

O bem-estar espiritual pode ser entendido como uma sensação de bem-estar que é experimentada quando encontramos um propósito que culmine num comprometimento com algo na vida, envolva um significado último para a vida e um sentimento de completude, de satisfação com a vida, paz com o mundo e consigo mesmo (numa visão expandida do eu, pessoa, self), de unidade e proximidade com um ser ou algo absoluto ou com a naturezå.

Bem-estar espiritual é um indicador de saúde espiritual9 . Uma extensa revisão sobre o tema apresenta que os principais domínios relacionados ao bem-estar espiritual referem-se a relação com: um outro transcendente, que envolve relações de fé, crenças, adoração interior e exterior, contemplação, percepção desse outro em sua vida (domínio transcendental); o self, referindo-se ao encontro de valores, significado, propósito e sentido para a vida (domínio pessoal); a comunidade, demonstrada por uma profunda disposição ao relacionamento interpessoal, relacionado com moralidade, cultura e religião (domínio social); e ambiente ou natureza, expresso pela disposição em cuidar do ambiente, reconhecer a natureza como fonte de vida e de se maravilhar com ela, podendo até sentir-se uno ou integrante dela (domínio ambiental) ${ }^{9}$.

Apesar de o termo espiritual não remeter diretamente a religiões, os estudos vêm mostrando que, desses quatro domínios, a relação com o outro transcendente, em particular com um deus, é a dimensão mais importante e que mais apresenta ou repercute em variações nas avaliações do bem-estar espiritual910. Essas evidências da repercussão da relação com o outro transcendente são, para as ciências da saúde, atualmente, um paradoxo que vem sendo explorado, confrontando questões metafísicas com indicadores objetivos em saúde.

A Escala de Bem-Estar Espiritual foi desenvolvida na década de 1980, com o objetivo de complementar os indicadores objetivos nas avaliações de qualidade de vida. Até hoje vários estudos internacionais vêm mostrando associações positivas entre o construto e indicadores de saúde ${ }^{8,11}$. 
A EBE é um instrumento subdividido em duas subescalas (de 10 itens cada), uma de Bem-Estar Religioso (BER) e outra de Bem-Estar Existencial (BEE). Os itens referentes ao BER contêm uma referência a Deus, e os de BEE referem-se à sensação de encontro com o sentido e o comprometimento com algo significativo na vida. Metade das questões da escala é escrita na direção positiva e metade, na negativa. A escala possui 20 questões que devem ser respondidas por meio de uma escala Likert de seis opções: Concordo Totalmente (CT), Concordo mais que discordo (Cd), Concordo Parcialmente (CP), Discordo Parcialmente (DP), Discordo mais que concordo (Dc) e Discordo Totalmente (DT). As questões com conotação positiva $(3,4,7,8,10,11,14,15,17,19$ e 20) têm sua pontuação somada da seguinte maneira: $C T=6, C d=5, C P=4, D P=3$, $D c=2$ e DT $=1$. As outras questões são elaboradas de forma negativa e devem ser somadas de forma invertida ( $C T=1, \mathrm{Cd}$ $=2, C P=3$, e assim por diante). $O$ escore total da escala, ou seja, o valor do Bem-Estar Espiritual é a soma das pontuações dessas 20 questões, podendo variar de 20 a 1208,9,11,12.

Embora haja outros instrumentos utilizados para avaliar a religiosidade e a espiritualidade e suas apresentações, a Escala de Bem-Estar Espiritual reflete em seus dois domínios duas questões peculiares do período de internamento hospitalar: a relação com Deus e as crises existenciais envolvidas. Considerando que a EBE não foi validada para ser aplicada à população de pacientes internados, em particular no período pré-operatório, no qual o paciente sofre de medo, angústia e incertezas que lhe prejudicam o bem-estar psicológico e subjetivo, há necessidade de estudar as propriedades psicométricas da versão brasileira nesse grupo. Além disso, a EBE avalia um estado, e o período pré-operatório revela-se como uma circunstância excepcional, em que os construtos avaliados pela escala podem se tornar mais evidentes.

Sendo assim, o presente estudo teve por objetivo validar o uso da Escala de Bem-Estar Espiritual para pacientes no período em internamento no pré-operatório.

\section{MÉTODOS}

Trata-se de um estudo de validação da versão brasileira da Escala de Bem-Estar Espiritual para aplicação em pacientes em internamento no período pré-operatório. O estudo foi realizado entre os meses de janeiro e abril de 2017, nas enfermarias de um hospital universitário especializado em cardiologia, referência regional em cirurgia cardíaca.

Os dados foram coletados utilizando-se um instrumento próprio contendo: um questionário elaborado para levantamento sociodemográfico, como sexo, idade, procedência, renda, escolaridade, afiliação religiosa, tipo de cirurgia, tempo de internamento etc.; além da escala.

A amostra teve por referência a meta de alcançar, minimamente, cinco respondentes por item, considerando que a
EBE possui 10 itens para fator, com significativa sobredeterminação que diminui a necessidade de amostras com valores maiores que 10 respondentes por item ${ }^{13}$.

Foram incluídos pacientes internados em período pré-operatório de cirurgia cardíaca de revascularização miocárdica, troca ou plastia valvar e excluídos os que apresentassem nível de consciência rebaixado, comunicação verbal prejudicada ou qualquer condição clínica ou psicológica que prejudicasse a entrevista ou a tornasse desconfortável; uso de antidepressivo; diagnóstico médico prévio referido de transtornos de humor, de ansiedade ou qualquer outro transtorno psiquiátrico.

Para cada paciente, foi aplicado o instrumento de coleta de dados descrito anteriormente, mediante entrevista. Foram realizadas 15 entrevistas piloto, de forma a familiarizar o entrevistador com o uso da escala. Incialmente, os participantes do estudo foram informados quanto aos procedimentos e objetivos da pesquisa e convidados à participação mediante a assinatura do Termo de Consentimento Livre e Esclarecido (TCLE), para em seguida serem realizadas as entrevistas e aplicado o instrumento de coleta.

As entrevistas foram realizadas à beira do leito, com anuência do enfermeiro responsável pelo setor no horário, bem como dos acompanhantes, que foram orientados a não intervir em nenhuma resposta se permanecessem ao lado dos pacientes durante a entrevista.

Os dados foram analisados com recursos de estatística descritiva e inferencial, e SPSS 24.0. Por meio do teste de Normalidade Kolmogorov-Smirnov, foi verificado que os desfechos avaliados apresentaram nenhuma distribuição normal $(<p=0,05)$. Os resultados da escala são apresentados com recursos de estatística descritiva ${ }^{13,14}$.

Com o objetivo de verificar a validade de construto, realizou-se análise fatorial exploratória utilizando o método de extração dos componentes principais (ACP) e rotação ortogonal do tipo Varimax. Foi verificada, pelo teste de esfericidade de Bartlett e pela medida de adequacidade da amostra de Kaiser-Meyer-Olkin (KMO), se o modelo era adequado à análise fatorial (Tabela 1). A ACP foi também explorada utilizando a análise paralela, para dirimir qualquer dúvida referente à quantidade de dimensões a serem extraídas, por ser mais robusta que os critérios de Kaiser e de Cattell, utilizados previamente. Tal análise compara os autovalores empíricos, encontrados na amostra, com autovalores médios de bancos de dados gerados aleatoriamente, com as mesmas características do banco empírico, ou seja, tamanho da amostra e número de itens do instrumento (matriz de mesmo tamanho). Ao comparar os autovalores, se o observado for maior que o simulado, há o apoio à existência do fator. O coeficiente alfa de Cronbach foi a medida utilizada para avaliar a fidedignidade da consistência interna dos fatores determinados pela análise fatorial. Por fim, a determinação da composição de cada um dos fatores foi baseada nas cargas fatoriais e, 
neste estudo, considerou-se como carga fatorial significante um valor de referência, em módulo, superior a 0,3, igual ao estipulado na validação inicial da versão brasileira ${ }^{8,15}$. Não se buscou avaliar a validade convergente, visto que não encontramos outra escala validada que refletisse tão bem os dois domínios da EBE (bem-estar religioso e existencial).

Tabela 1. Avaliação dos parâmetros da análise fatorial para a Escala de Bem-Estar Espiritual aplicado a pacientes internados em período pré-operatório - Recife-PE, Brasil, 2017

\begin{tabular}{lcc}
\hline Parâmetros de adequacidade & Valores ideais & Valores observados \\
\hline KM0 & $\geq 0,60$ & 0,87 \\
Teste de esfericidade de Bartlett $(\mathrm{p})$ & $<0,05$ & $<0,001$ \\
Alfa de Cronbach & $\geq 0,50$ & $0,66-0,89$ \\
Autovalores dos fatores & $\geq 1,0$ & $0,11-10,46$ \\
Total da variância explicada - 2 fatores & $\geq 50 \%$ & $43,33 \%$ \\
Carga fatorial das questões & $>0,40$ & $0,20-0,88$ \\
Comunalidade & $\geq 0,50$ & $0,15-0,77$ \\
\hline
\end{tabular}

A pesquisa foi elaborada pautada nos preceitos da Resolução CNS no 466/2012. O início da coleta de dados deu-se apenas após a apreciação e aprovação do Comitê de Ética em Pesquisa do Pronto-Socorro Cardiológico Universitário de Pernambuco (Procape/UPE) - Parecer no 717,788, CAAE: 30622414.7.0000.5192.

\section{RESULTADOS}

Participaram do estudo 174 pacientes, com distribuição de gênero bem equilibrada (50,6\% homens, $49,4 \%$ de mulheres); $54,6 \%$ eram casados ou com companheiro, de religião católica $(55,2 \%)$ ou evangélica $(44,8 \%)$ e proveniente do interior do estado (49,1\%). A média de idade foi de 59,16 \pm 13,86 anos; 55,7\% tinham idade acima de 60 anos.

A maior parte da amostra afirmou-se sem atividade laboral $(66,7 \%)$, tendo apenas um terço deles ativos no mercado de trabalho (33,3\%). A renda média observada entre eles é de 1,31 \pm 0,96 salários mínimos, e 70,7\% declararam receber até um salário.

Apenas um pouco mais de um quinto da amostra (22,5\%) já havia sido submetido a uma cirurgia cardíaca prévia. Em média, os pacientes estavam internados por 22,06 $\pm 11,08$ dias, e 70,7\% mais do que 15 dias; o período médio de pré-operatório foi igual a 20,95 $\pm 29,78$, com $56,9 \%$ dos pacientes em até 15 dias, e 43,1\%, com período superior a 15 dias. Para a grande maioria dos pacientes, houve um a presença de um acompanhante $(85,6 \%)$ e visita religiosa $(65,5 \%)$, porém uma visita diária não foi observada tão frequentemente (25,3\%). Apenas em 6,9\% dos casos houve uma solicitação de visita religiosa.
A amostra com 174 participantes continha a razão de 8,7 pessoas por item (variável), considerada adequada ao propóstico $(K M O=0,87)$ e o Teste de Esferacidade de Bartlett's foi significativo $\left(\chi^{2}=1401,67 ; p<0,01\right)$, revelando que há relações significativas entre as variáveis e que a análise fatorial é apropriada13 (Tabela 1).

A extração de componentes principais, realizada com o objetivo de explorar a estrutura dos dados, mostrou que o primeiro e o segundo componentes detinham autovalores superiores a 2,0 e, juntos, explicavam 43,33\% da variância total. Numa simulação da rotação em três fatores, verificou-se que não haveria incremento para mais de 50\% na variância explicada pelo modelo. Numa observação do gráfico do scree (Figura 1), verifica-se que a partir do terceiro componente os autovalores decrescem e se apresentam como se houvesse uma variância comum, sugerindo que os 20 itens da EBE deveriam estar organizados em dois componentes, como encontrado na validação original da escala.

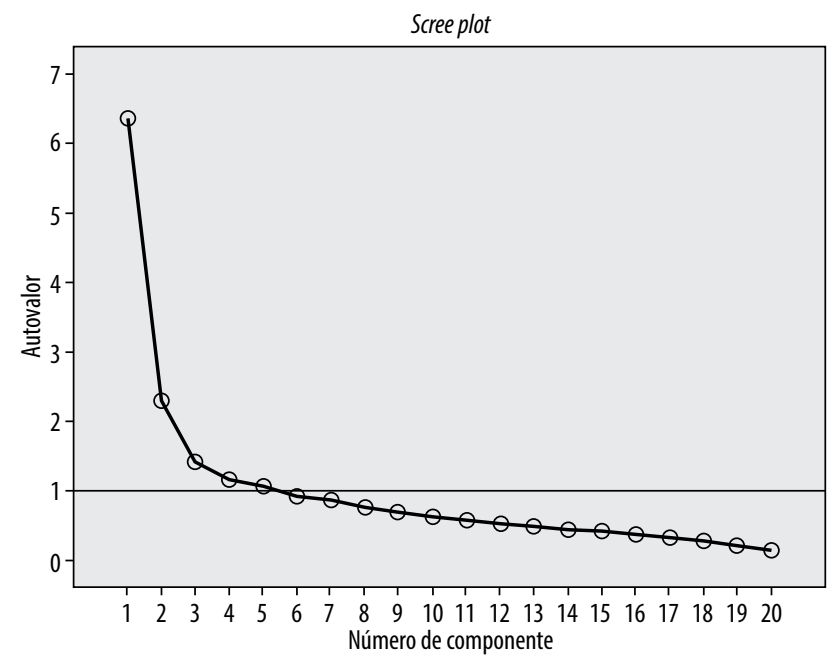

Figura 1. Scree plot dos autovalores da Escala de Bem-Estar Espiritual.

A análise paralela revelou dois componentes com autovalores que excederam o correspondente estimado em uma matriz aleatória de mesmo tamanho (20 variáveis $\times 174$ respondentes). A partir do terceiro, mesmo que ainda maior que 1 , o eigenvalue encontrado era menor que o estimado $(1,42 \times 1,44)$.

Para proceder ao exame da validade de construto da EBE, foi realizada a análise fatorial por meio do método de extração dos eixos principais (Principal Axis Factoring - PAF) com rotação Varimax, sendo realizada a extração de dois fatores, relacionados às subescalas da versão validada da $\mathrm{EBE}^{8}$. Foi escolhida a rotação ortogonal no intuito de confirmar a escoIha dos autores que validaram a escala no Brasil, considerando que o construto das duas subescalas é distinto. Os dois 
fatores rotacionados ficaram constituídos como a proposta inicial, com os itens ímpares representando a subescala de Bem-Estar Religioso, com valores significativos para o fator 1 e $a=0,84$, e os pares representando a subescala de Bem-Estar Existencial, com o fator 2 e $a=0,71$. O nível de correlação entre os dois fatores foi de 0,47 .

Tabela 2. Carga fatorial (CF), média (Md), desvio-padrão (dp) e comunalidades (h2) dos itens da Escala de Bem-Estar Espiritual - Recife-PE, Brasil, 2017

\begin{tabular}{lccccccc}
\hline & CF & Md (dp) & h2 & & CF & Md (dp) & h2 \\
\hline EBE 17 & 0,88 & $5,7(0,9)$ & 0,77 & EBE 20 & 0,56 & $5,7(0,7)$ & 0,31 \\
EBE 11 & 0,84 & $5,7(0,8)$ & 0,72 & EBE 8 & 0,53 & $5,1(1,4)$ & 0,51 \\
EBE 19 & 0,82 & $5,8(0,8)$ & 0,69 & EBE 4 & 0,50 & $5,2(1,4)$ & 0,37 \\
EBE 15 & 0,75 & $5,8(0,9)$ & 0,60 & EBE 12 & 0,44 & $5,0(1,7)$ & 0,24 \\
EBE 7 & 0,74 & $5,7(0,9)$ & 0,57 & EBE 10 & 0,44 & $4,6(1,6)$ & 0,69 \\
EBE 5 & 0,64 & $5,5(1,3)$ & 0,45 & EBE 18 & 0,41 & $4,7(1,9)$ & 0,20 \\
EBE 3 & 0,64 & $5,8(0,9)$ & 0,46 & EBE 14 & 0,31 & $4,7(1,4)$ & 0,47 \\
EBE 9 & 0,53 & $5,3(17)$ & 0,37 & EBE 2 & 0,27 & $3,7(2,1)$ & 0,25 \\
EBE 1 & 0,45 & $5,1(1,7)$ & 0,30 & EBE 6 & 0,23 & $3,8(1,9)$ & 0,26 \\
EBE 13 & 0,41 & $4,9(1,8)$ & 0,28 & EBE 16 & 0,20 & $3,0(1,9)$ & 0,15 \\
\hline
\end{tabular}

A análise das cargas fatoriais para a subescala de Bem-Estar Religioso apresentou distribuição próxima à da validação inicial, com todos os itens com carga fatorial >0,3. Na avaliação dos itens da subescala de Bem-Estar Existencial, três itens alcançaram escores menores que 0,3 de carga fatorial. A carga fatorial abaixo de 0,3 caracteriza que não houve forte correlação entre esses itens e o fator correspondente, sugerindo que esses itens ( 2,6 e 16$)$ poderiam ser retirados da escala.

$\mathrm{Na}$ avaliação do bem-estar espiritual, considerando as duas dimensões avaliadas pela escala - o bem-estar existencial e o religioso -, foi possível verificar que o bem-estar existencial teve valor médio inferior ao observado para o religioso $(45,62 \pm 8,69 ; 55,30 \pm 8,08$, respectivamente). O bem-estar total apresentou valor médio elevado $(100,93 \pm 14,07)$. Ambas as dimensões do bem-estar espiritual (religioso e existencial) tiveram valor aceitável para o índice de confiabilidade $(a=0,84 ; a=0,71)$, indicando boa consistência interna. No total, a Escala de Bem-Estar Espiritual apresentou boa confiabilidade $(a=0,79)$. Não existe correlação forte entre os itens e, na análise dos itens, a retirada de nenhum deles incrementaria significativamente o desempenho da subescala correspondente (Tabela 3).

Tabela 3. Estatísticas dos itens de bem-estar religioso e existencial - Recife-PE, Brasil, 2017

\begin{tabular}{|c|c|c|c|c|c|}
\hline \multicolumn{6}{|c|}{ Estatísticas de item-total - subescala Bem-Estar Religioso } \\
\hline & $\begin{array}{l}\text { Média de escala se o item for } \\
\text { excluído }\end{array}$ & $\begin{array}{c}\text { Variância de escala se o item for } \\
\text { excluído }\end{array}$ & $\begin{array}{l}\text { Correlação de item total } \\
\text { corrigida }\end{array}$ & Correlação múltipla ao quadrado & $\begin{array}{l}\text { Alfa de Cronbach se o item for } \\
\text { excluído }\end{array}$ \\
\hline EBE 1 & 50,21 & 51,02 & 0,45 & 0,23 & 0,85 \\
\hline EBE 3 & 49,53 & 57,35 & 0,52 & 0,43 & 0,83 \\
\hline EBE 5 & 49,75 & 52,27 & 0,62 & 0,42 & 0,82 \\
\hline EBE 7 & 49,63 & 55,43 & 0,63 & 0,56 & 0,83 \\
\hline EBE 9 & 50,05 & 48,93 & 0,56 & 0,40 & 0,83 \\
\hline EBE 11 & 49,55 & 55,85 & 0,71 & 0,74 & 0,82 \\
\hline EBE 13 & 50,39 & 50,46 & 0,44 & 0,29 & 0,85 \\
\hline EBE 15 & 49,53 & 55,57 & 0,65 & 0,57 & 0,82 \\
\hline EBE 17 & 49,60 & 55,48 & 0,71 & 0,73 & 0,82 \\
\hline EBE 19 & 49,51 & 55,84 & 0,73 & 0,64 & 0,82 \\
\hline \multicolumn{6}{|c|}{ Estatisticas de item-total - subescala Bem-Estar Existencial } \\
\hline & $\begin{array}{c}\text { Média de escala se o item for } \\
\text { excluído }\end{array}$ & $\begin{array}{c}\text { Variância de escala se o item for } \\
\text { excluído }\end{array}$ & $\begin{array}{l}\text { Correlação de item total } \\
\text { corrigida }\end{array}$ & Correlação múltipla ao quadrado & $\begin{array}{l}\text { Alfa de Cronbach se o item for } \\
\text { excluído }\end{array}$ \\
\hline EBE 2 & 41,90 & 60,69 & 0,32 & 0,20 & 0,69 \\
\hline EBE 4 & 40,46 & 63,81 & 0,44 & 0,25 & 0,67 \\
\hline EBE 6 & 41,82 & 60,95 & 0,35 & 0,17 & 0,68 \\
\hline EBE 8 & 40,49 & 62,78 & 0,51 & 0,32 & 0,66 \\
\hline EBE 10 & 41,01 & 57,88 & 0,59 & 0,46 & 0,64 \\
\hline EBE 12 & 40,58 & 67,99 & 0,26 & 0,17 & 0,72 \\
\hline EBE 14 & 40,90 & 63,84 & 0,42 & 0,39 & 0,67 \\
\hline EBE 16 & 42,54 & 62,92 & 0,30 & 0,20 & 0,69 \\
\hline EBE 18 & 40,94 & 60,65 & 0,37 & 0,19 & 0,68 \\
\hline EBE 20 & 39,96 & 70,95 & 0,34 & 0,15 & 0,69 \\
\hline
\end{tabular}




\section{DISCUSSÃO}

Ansiedade, depressão e medo são construtos que comumente já foram evidenciados em elevados escores no período pré-operatório e que comprometem o bem-estar subjetivo ${ }^{16,17}$. No entanto, a dimensão espiritual da pessoa que enfrenta adoecimento, internamento e cirurgia tem sido pouco relatada, devendo ser validados para essa população os instrumentos disponíveis para avaliação da espiritualidade, religiosidade e seus desdobramentos e expressões.

Na validação para o Brasil da escala, nenhum item das subescalas teve valor abaixo do corte para a carga fatorial, e os autores não sugeriram alteração da escala inicial ${ }^{8}$. No nosso estudo, apesar da carga fatorial abaixo de 0,3 para dois itens, sugerindo que eles $(2,6$ e 16) poderiam ser retirados da escala, a análise mostrou que não haveria incremento no desempenho da escala essa mudança, optando-se por não retirá-los.

Para essa amostra, a aplicação da escala teve, em média, duração de 8,42 \pm 4,62 minutos, maior que no estudo de referência da validação para o português ${ }^{8}$. Esse fato pode estar relacionado com a escolaridade menor, assim como com a dificuldade em clarear aos pacientes a forma de avaliar cada item, atribuindo-lhes valor.

A despeito da boa fidedignidade da subescala, a carga fatorial menor para a maioria dos itens do Bem-Estar Existencial pode ter-se dado pela dificuldade de compreensão das proposições, visto que as elas sugerem questões que requerem nível maior de abstração e subjetividade, que se tornam dif́ceis de se responderem com precisão por uma escolha de valoração por meio de uma escala tipo Likert com seis opções. Além disso, por se tratar de uma questão cultural fortemente predominante, os pacientes apresentam, de forma geral, uma religiosidade que lhes traz respostas para as possíveis crises espirituais que possam ter necessidade de enfrentar.

Deve-se considerar que, de certa forma, para pacientes religiosos, a alternância de itens que se referem a deus, com itens de reflexões existenciais, pode influenciar para uma escolha de respostas mais positivas, como poderia ocorrer ao contrário em amostras pouco religiosas. Por isso, para uma avaliação acerca da espiritualidade, sugere-se ainda o uso de outras escalas que não tenham domínios relacionados diretamente a um deus ou, como foi sugerido no estudo de validação nacional, em que possa ser substituída a palavra deus por uma força superior, algo transcendente ou outra forma que contemple a crença do sujeito?.

\section{CONCLUSÃO}

Avaliar o bem-estar espiritual dos pacientes durante o internamento hospitalar é de suma importância para a abordagem da pessoa como um todo, devendo ser aprimorados os recursos disponíveis para tal.
Ainda que a amostra tenha sido adequada, como limitação do estudo consideramos que uma amostra maior que dez respondentes por item poderia descrever de forma mais próxima do real o comportamento da escala.

A Escala de Bem-Estar Espiritual mostrou-se possível de ser aplicada entre pacientes internados no período pré-operatório, útil para avaliar o construto a que se propõe, mesmo sem ter apresentado o mesmo desempenho do estudo de validação, realizado na população em geral não doente. A subescala Bem-Estar Existencial apresentou desempenho menor em relação à subescala Bem-Estar Religioso.

Por fim, sugere-se a adaptação dessa escala para essa população, com redação mais simples e adequada a baixa escolaridade e aspectos culturais, para facilitar e aperfeiçoar a mensuração desses construtos.

\section{CONTRIBUIÇÕES INDIVIDUAIS}

Eduardo Tavares Gomes - Contribuiu significativamente na concepção e desenho do estudo, coleta, na análise e interpretação dos dados, redação do artigo, na revisão crítica relevante do conteúdo intelectual e na aprovação da versão final a ser publicada.

Simone Maria Muniz da Silva Bezerra - Contribuiu significativamente na concepção e desenho do estudo, na análise e interpretação dos dados, na redação do artigo, na revisão crítica relevante do conteúdo intelectual e na aprovação da versão final a ser publicada.

\section{CONFLITOS DE INTERESSE}

Os autores Eduardo Tavares Gomes e Simone Maria Muniz da Silva Bezerra não possuem conflitos de interesse a serem declarados. Não houve suporte financeiro para a pesquisa.

\section{REFERÊNCIAS}

1. Kalichman A0, Ayres JRCM. Integralidade e tecnologias de atenção à saúde: uma narrativa sobre contribuições conceituais à construção do princípio da integralidade no SUS. Cad Saúde Pública. 2016;32(8):e00183415.

2. Panzini RG, Rocha NS, Bandeira DR, Fleck MPA. Qualidade de vida e espiritualidade. Arch Clin Psychiatry. 2007;34(Supl 1):105-15.

3. Salgado MRC, organizadora. Saúde integral: uma interação entre ciência e espiritualidade. São Paulo: AME Brasil; 2013. 260p.

4. Santos ARM, Dabbisco P, Cartaxo HGO, Silva EAPC, Souza MRM, Freitas CMSM. A systematic review of the influence of religiosity on the adoption of an active lifestyle. Rev Bras Promoc Saude. 2013;26(3):419-25.

5. Aquino TAV. A presença não ignorada de Deus na obra de Vitor Frankl. São Paulo: Paulus; 2014

6. Watson J. Intentionality and caring-healing consciousness: a practice of transpersonal nursing. Holist Nurs Pract. 2002;16(4):12-9. 
7. Watson J. Watson's theory of human caring and subjective living experiences: carative factors/caritas processes as a disciplinary guide to the professional nursing practice. Texto Contexto Enferm. 2007;16(1):129-35.

8. Marques LF, Sarriera JC, Dell'Aglio DD. Adaptação e validação da Escala de Bem-estar Espiritual (EBE). Aval Psicol. 2009;8(2):179-86.

9. Gomez R, Fisher JW. Domains of spiritual well-being and development and validation of the Spiritual Well-Being Questionnaire. Personality and Individual Differences. 2013;35:1975-91.

10. Fisher J. Assessing spiritual well-being: relating with God explains greatest variance in spiritual well-being among Australian youth. Int J Children's Spirituality. 2013;18(4):306-17.

11. Silva RPS, Souza P, Nogueira DA, Moreira DS, Chaves ECL. Relação entre bem-estar espiritual, características sociodemográficas e consumo de álcool e outras drogas por estudantes. J Bras Psiquiatr. 2013;62(3):191-8.
12. Volcan SMA, Sousa PL, Mari IJJ, Horta BL. Relação entre bem-estar espiritual e transtornos psiquiátricos menores: estudo transversal. Rev Saúde Pública. 2006;37(4):440-5.

13. Damásio BF. Uso da análise fatorial exploratória em psicologia. Aval Psicol. 2012;11(2):213-28.

14. Pasquali L. Psicometria. Rev Esc Enferm USP. 2009;43(Esp):992-9.

15. Pereira AA, Santos SME, Faria RMD. Brazilian version of the Attribution Questionnaire - Cross cultural adaptation and validation of psychometric properties. J Bras Psiquiatr. 2016;65(4):314-21

16. Gomes ET, Bezerra SMMS. Ansiedade e depressão no período pré-operatório de cirurgia cardíaca. Rev RENE. 2017;18:420-7.

17. Gonçalves KKN, Silva Jl, Gomes ET, Pinheiro LLS, Figueiredo TR, Bezerra SMMS. Anxiety in the preoperative period of heart surgery. Rev Bras Enferm. 2016;69(2):374-80. 